\title{
Development of an Optical Waveguide Interferometric Immunosensor
}

\author{
R. G. HEIDEMAN, R. P. H. KOOYMAN and J. GREVE
}

University of Twente, Department of Applied Physics, P.O. Box 217, NL-7500 AE Enschede (The Netherlands)

\begin{abstract}
A Mach-Zehnder interferometer is presented, which uses the evanescent field of a planar optical waveguide as the sensing element. Changes in the refractive index profile occurring in the immediate vicinity of the waveguide surface cause the propagation velocity of the light passing through it to change. This is measured interferometrically. Adsorption processes of antibodies onto the waveguide surface as well as immunoreactions have been monitored. Concentrations of $2.5 \times 10^{-8} \mathrm{M}\left(1 \times 10^{-3} \mathrm{mg} / \mathrm{ml}\right)$ of the antigen HCG (molecular weight $\approx 39600$ ) have been measured so far.
\end{abstract}

\section{Introduction}

The exploitation of the evanescent field built up in an optical waveguide structure has become popular in the development of immunosensors [1]. Here, the selector molecule (the antibody) is immobilized onto the waveguide surface. A specific reaction with its partner molecule (the analyte) results in the growth of an additional layer. This can be considered as a changing refractive index profile in the immediate vicinity of the waveguide surface, within the evanescent field. The waveguide structure is accordingly changed, which can be measured by a variety of optical methods [2].

By using an interferometric set-up, the change in the propagation velocity of the light passing through the waveguide is detected. The combination of interferometric detection and relatively long interaction lengths gives rise to a potentially very sensitive sensor. It can be used for all kinds of biological experiments which involve a changing refractive index profile.

\section{Materials and Methods}

In order to get the sensor to be as sensitive as possible, the propagation velocity of the light travelling through the waveguide must depend steeply on the refractive index profile near the surface. This can be achieved by making the difference between the refractive indices of the actual waveguiding layer and the substrate as large as possible. Furthermore, the thickness of the waveguiding layer must be approximately twice the cut-off thickness [3]. In our experiments we used the waveguide structure shown in Fig. 1.

The waveguide structure is fabricated using standard techniques. We start with a 2 inch wafer, onto which we thermally grow an $\mathrm{SiO}_{2}$ layer (refractive index of 1.457) with a thickness of approximately $1 \mu \mathrm{m}$. This layer acts as the substrate, on top of which the actual waveguiding layer of $\mathrm{Si}_{3} \mathrm{~N}_{4}$ is deposited using low pressure chemical vapour deposition. This $\mathrm{Si}_{3} \mathrm{~N}_{4}$ layer has a refractive index of 2.0 and a thickness of $\approx 0.1 \mu \mathrm{m}$. On top of this, a protective $1 \mu \mathrm{m} \mathrm{SiO}_{2}$ layer is grown using plasma enhanced chemical vapour deposition. This protective layer is completely removed (see Fig. 1) in two rectangles with a length of $1.0 \mathrm{~cm}$ (interaction length). This is done with wet chemical etching using a $\mathrm{HF} / \mathrm{NH}_{4} \mathrm{~F}$ solution. In the same way, two regions with a grating are fabricated in the $\mathrm{SiO}_{2}$ layer. These gratings are used to couple light into and out of the waveguide. The grating periods are $4 \mu \mathrm{m}$

(C) Elsevier Sequoia/Printed in The Netherlands 


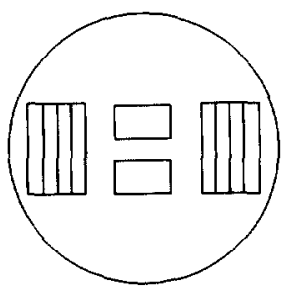

(a)

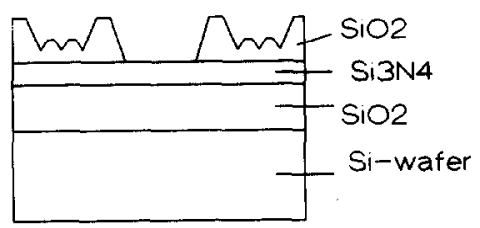

(b)

Fig. 1. The waveguide structure used. (a) Top view; (b) cross section.

and $2 \mu \mathrm{m}$ for the in- and outcoupling gratings respectively.

The waveguide structure is placed in an interferometric set-up (see Fig. 2).

A laser beam ( $\mathrm{He}-\mathrm{Ne}$ laser, wavelength $0.633 \mu \mathrm{m}$ ) is split into two parallel beams by the first cubic beamsplitter. Both beams are coupled into and out of the waveguide structure using the two grating couplers described above. A second beamsplitter is used to recombine both beams, resulting in two interference patterns. A rotatable glass slide is placed in one of the arms of the interferometer. Between the two grating couplers, a twochannel flow-through cuvette is pressed to the waveguide on top of the protective $\mathrm{SiO}_{2}$ layer.

The intensities of both interference patterns are measured with photodiodes. The difference between the two intensities is regulated to zero by rotating the glass slide using a feedback loop. The regulation current is used as the signal, providing a linearized phase change as the interferometer output, a constant sensitivity and a decreased intensity dependence.

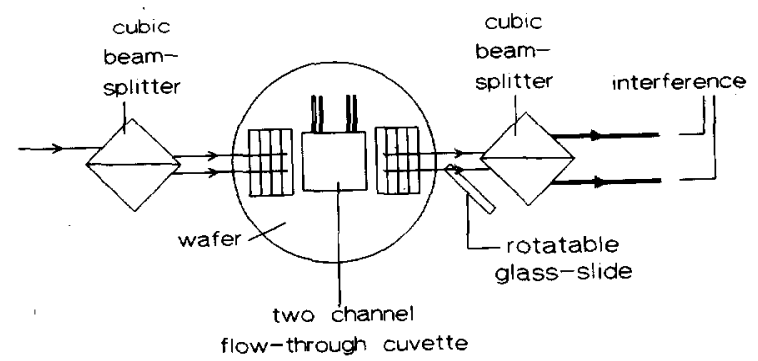

Fig. 2. Set-up for the waveguide interferometer.

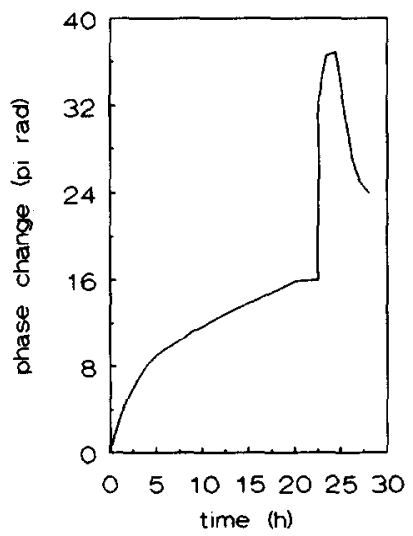

(a)

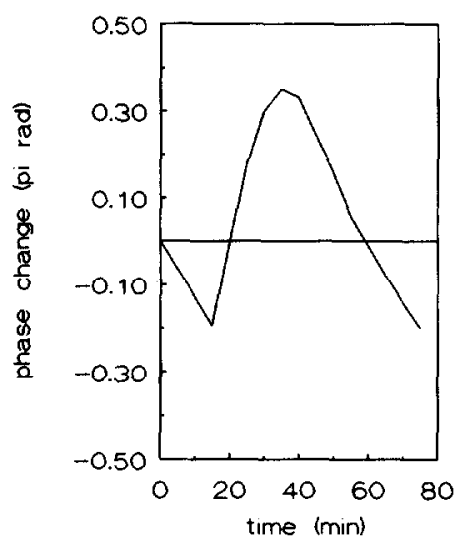

(b)

Fig. 3. Immunoreaction experiment. (a) $t=0.0 \mathrm{~h}$ : the antibody protein $\alpha \mathrm{HCG}$ is pumped through (molar concentration $\approx 3 \times 10^{-8}$ ). $t=22.5 \mathrm{~h}$ : the protein BSA (molar concentration $\approx 1.5 \times 10^{-4}$ ) is pumped through. $t=24.5 \mathrm{~h}$ : Sörensen buffer is pumped through. (b) $t=15 \mathrm{~min}$ : the antigen protein $\mathrm{HCG}$ is pumped through (molar concentration $\approx 2.5 \times 10^{-8}$ ), resulting in an immunoreaction, while the protein BSA is still being washed off the waveguide surface. 


\section{Results}

In the immunoreaction experiments, first both channels of the flow-through cuvette are filled with a buffer solution (Sörensen buffer). This buffer solution is continuously pumped through both channels at a rate of $\approx 0.3 \mathrm{ml}$ per minute, using a closed vessel system. The drift in the signal during this period should ideally be zero, but is $\approx 0.06 \pi$ in practice. Then the same buffer solution, but now with a dissolved antibody protein, is pumped through in one channel, resulting in an adsorption process. When the adsorption process is nearly completed, a new solution with a high concentration of the protein BSA is pumped through this channel. This is done to make sure that there are no empty places left at the surface of the waveguide so that the antigen protein cannot adsorb at the waveguide surface. Then again the buffer solution is pumped through until the signal is more or less stable. Finally, the buffer solution containing the antigen protein is pumped through, resulting in an immunoreaction.

A typical measurement is shown in Fig. 3. The concentration of the antibody protein $\alpha \mathrm{HCG}$ is $5 \times 10^{-8} \mathrm{M}$ (molecular weight $\approx 150000$ ). The concentration of the protein BSA is $\approx 1.5 \times 10^{-4} \mathrm{M}$ (molecular weight $\approx 68000$ ). The concentration of the antigen HCG is $2.5 \times 10^{-8} \mathrm{M}$ (molecular weight $\approx 39600$ ).

\section{Discussion and Conclusions}

The growth of a layer of proteins can be very sensitively detected using an interfero- metric detection scheme in combination with a planar waveguide. We detected the adsorption of several types of antibodies in different concentrations. Also immunoreactions with the antigen HCG down to a concentration of $2.5 \times 10^{-8} \mathrm{M}$ have been measured, resulting in a phase change of $\approx 0.6 \pi$. The measuring resolution is determined by the drift in the phase change, which is $\approx 0.06 \pi$ per hour during the experiments. The drift can be further minimized using a more rigid set-up.

\section{Acknowledgements}

These investigations in the program of the Foundation for Fundamental Research on Matter (FOM) have been supported (in part) by the Netherlands Technology Foundation (STW).

The gratings are fabricated with the aid of a grating mask. For the use of this mask, we would like to thank Dr P. V. Lambeck, Electronics Department, University of Twente, and Dr A. H. Verbruggen, Center for Submicron Technology (CST), University of Delft.

\section{References}

1 J. F. Place, R. M. Sutherland and C. Dahne, Optoelectronic immunosensors: a review of optical immunossay at continuous surfaces, Biosensors, 1 (1985) 321-353.

2 R. E. Dessy, Waveguides as chemical sensors, Anal. Chem., 61 (1989) 1079a-1094a.

$3 \mathrm{~K}$. Tiefentahler and W. Lukosz, Sensitivity of grating couplers as integrated-optical chemical sensors, $J$. Opt. Soc. Am. B, 6(1989) 209-220. 